\title{
From Macromolecules to Cells: Progress and Challenges in Mesoscale Electron Tomography
}

Barbara L. Armbruster*, Masahiro Kawasaki*, Jaap Brink*, Thomas Isabell*, Robert O’Donneli*, H. Furukawa**, M. Shimizu** and Michael Kersker*

*JEOL USA, Inc. Peabody, MA 01960 USA

**JEOL System Technology Company Ltd, Akishima, Japan

Electron tomography and cryotomography provide 3D views of resin-embedded or frozenhydrated cells, respectively, to reveal macromolecular complexes in the spatial context in which they normally function. The spatial resolution in a tomogram is currently limited to approximately $3 \mathrm{~nm}$ for either resin embedded or frozen-hydrated biological specimens given the geometry limitations that the stage imposes on data acquisition. Yet, hybrid methods allow for the extraction of much higher resolution information. Tomographic slices along the projection direction in the microscope are superior in resolution to any physically sectioned cell and they are free of distortions thus revealing new details in mitochondrial structure and function [1]. Marsh et al's investigations of the convoluted structure of the Golgi in insulinoma cells [2] demonstrated details in structural connectivity that could not be consistently observed in serial section reconstructions.

Despite these advances, many obstacles still remain as the resolution and interpretation is pushed towards the $1 \mathrm{~nm}$-level on the one hand as well as towards encompassing structures much larger than the originally reconstructed object. At this mesoscale the challenges for researchers are formidable. Many new tools are available to investigators. For instance, autotracking and/or alignment without fiducial markers, as employed in software programs such as JEOL's TEMography ${ }^{\mathrm{TM}}$ [3], and freeware solutions such as SerialEM (University of Colorado in Boulder [4]), and Mr. T (Baylor College of Medicine [5]), allow for an effective minimization of the electron dose on the specimen. TEMography ${ }^{\mathrm{TM}}$ is unique as it constitutes a turn-key solution, comprised of three independent modules: Recorder to acquire a tilt series (Fig. 1), Composer to reconstruct the data from a tilt series (Fig. 2), and Visualizer to visualize the reconstructed data with integrated surface, volume and slice rendering capabilities (Fig. 3). Sophisticated alignment and reconstruction algorithms take over repetitive optimization steps. Automated most-probable loss tomography for thick sections [6] dramatically improves the resolution in projections by decreasing the contribution of inelastic scattering in the dataset. Energy filtered imaging offers contrast enhancement in the zero-loss mode or elemental distribution in spectrum imaging or single element mapping modes [7]. Autosegmentation algorithms are needed to quickly construct interpretable threedimensional volumes from tomograms. The new generation of aberration-corrected microscopes equipped with phase plates and large-area detectors promises tomograms with higher resolution and better contrast to overcome mesoscale challenges.

[1] Manella, C.A., Biochim. Biophys. Acta., 1762 (2006) 140.

[2] Marsh, B.J., Biochim. Biophys Acta 1744 (2005) 273.

[3] Motoki, S., et al., Proc. 8 Asia Pacific Elec. Microsc. Conf., Kanazawa, Japan (2004) 178.

[4] Mastronarde, D.N., J. Struct. Biol. 152 (2005) 36.

[5[ Chang, J., et al., Microsc. Microanal., 11(2005 Spl. 2) 308CD.

[6] Bouwer, J.C., et al., J. Struct. Biol., 148 (2004) 297.

[7] Marko, M., et al., Microscopy Today, 11 (2002)14. 


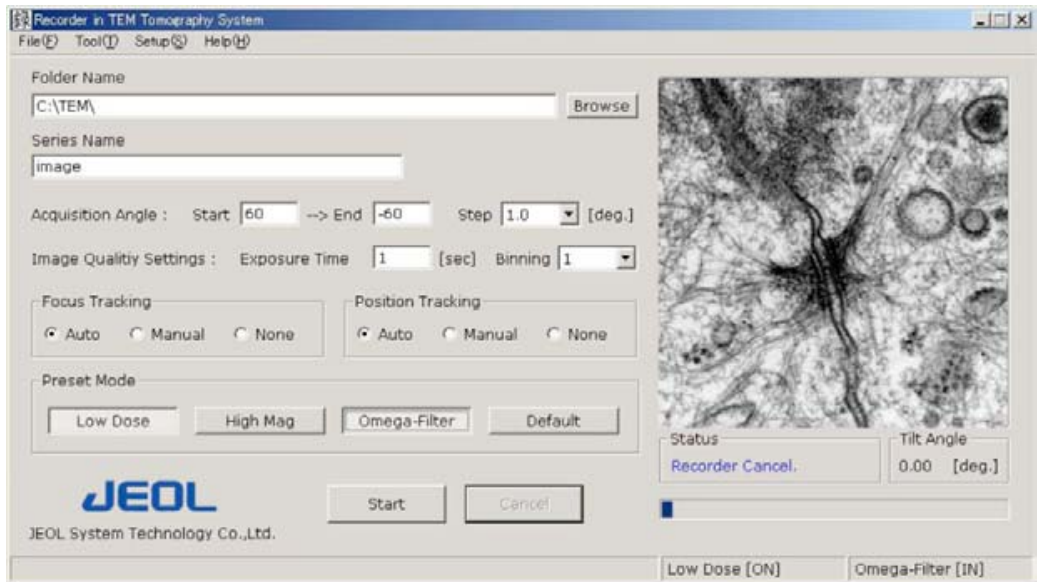

Figure 1. Recorder allows the user to set parameters for image acquisition including step size, focus and position tracking, as well as low dose, high magnification and omega-filtered imaging.

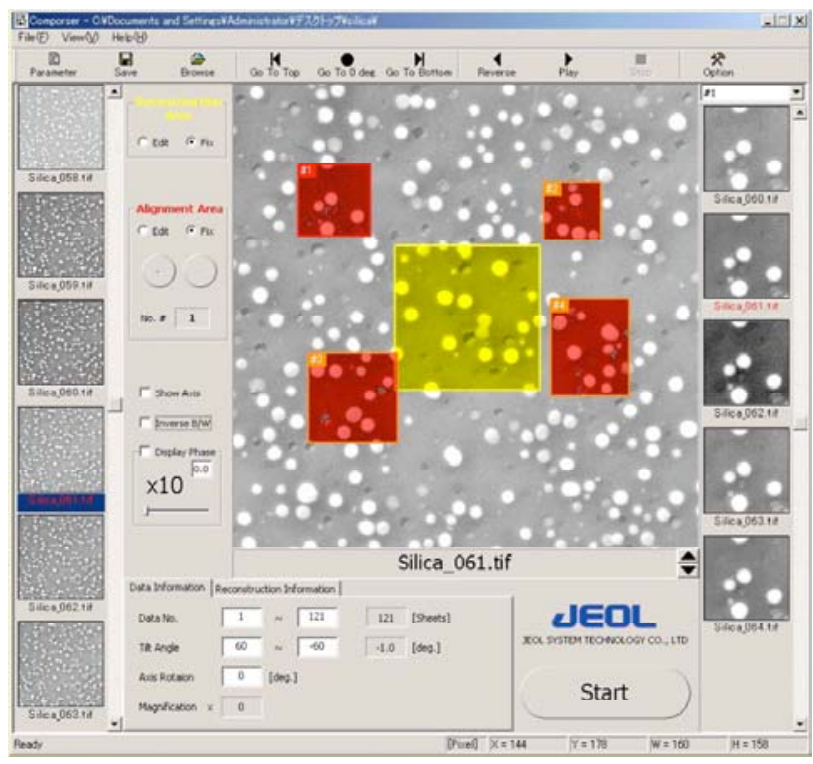

Figure 2: Composer allows the user to set parameters for aligning the raw tilt series data and to view/correct alignment results and to reconstruct the tilt series data.

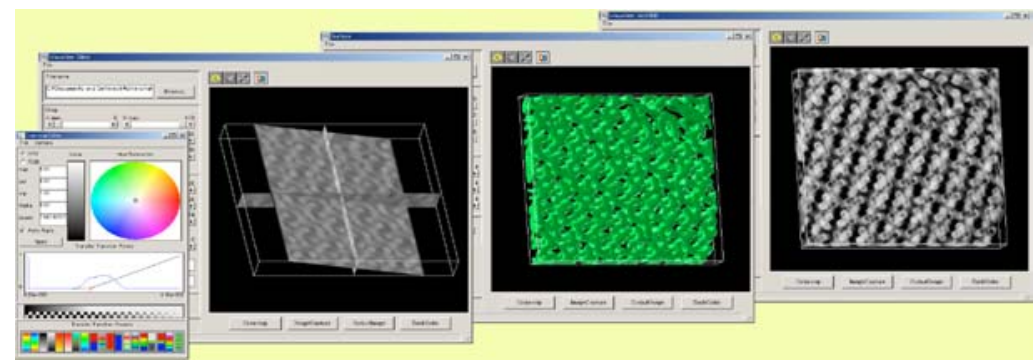

Figure 3. The Visualizer package supports multiple simultaneous cross-section views in arbitrary directions, volume and surface rendering, and flexible color mapping and highlighting. 